\title{
Extended elliptic-type integrals with associated properties and Turán-type inequalities
}

\author{
Rakesh K. Parmar ${ }^{1}$, Ritu Agarwal ${ }^{2}$, Naveen Kumar $^{2 *}$ and S.D. Purohit ${ }^{3}$
}

${ }^{*}$ Correspondence:
naveenahrodia@gmail.com
${ }^{2}$ Department of Mathematics,
Malaviya National Institute of
Technology, Jaipur, 302017, India
Full list of author information is
available at the end of the article

\begin{abstract}
Our aim is to study and investigate the family of $(p, q)$-extended (incomplete and complete) elliptic-type integrals for which the usual properties and representations of various known results of the (classical) elliptic integrals are extended in a simple manner. This family of elliptic-type integrals involves a number of special cases and has a connection with $(p, q)$-extended Gauss' hypergeometric function and $(p, q)$-extended Appell's double hypergeometric function $F_{1}$. Turán-type inequalities including log-convexity properties are proved for these $(p, q)$-extended complete elliptic-type integrals. Further, we establish various Mellin transform formulas and obtain certain infinite series representations containing Laguerre polynomials. We also obtain some relationship between these $(p, q)$-extended elliptic-type integrals and Meijer G-function of two variables. Moreover, we obtain several connections with $(p, q)$-extended beta function as special values and deduce numerous differential and integral formulas. In conclusion, we introduce $(p, q)$-extension of the Epstein-Hubbell (E-H) elliptic-type integral.
\end{abstract}

MSC: Primary 33B15; 33C05; 33E05; 33C65; secondary 33B99; 33C60; 33C75

Keywords: Turán-type inequalities; Elliptic integrals; Extended beta function; Extended hypergeometric functions; Mellin transform; Laguerre polynomials

\section{Introduction, preliminaries, and motivation}

Elliptic-type integrals such as (classical) Legendre elliptic integrals, generalized complete elliptic integrals of the first and second kind (see, for example, [5, 29, 30, 33-35]), and symmetric elliptic integrals [7] and also several definite integrals of such families are known to play a prominent role in special functions in terms of their modulus or complementary modulus in the theory of conformal mappings [2], studies of crystallographic minimal surfaces, radiation physics problems [3], nuclear technology, fracture mechanics studies of elliptical crack problems, the study of electromagnetic or acoustic waves being scattered by an elliptic disk [15], astronomy, geometry, physics, and engineering mechanics [4]. In recent years (2009), Lin et al. [16, p. 1178, Eq. (1.12)] introduced and extensively

(c) The Author(s) 2021. This article is licensed under a Creative Commons Attribution 4.0 International License, which permits use, sharing, adaptation, distribution and reproduction in any medium or format, as long as you give appropriate credit to the original author(s) and the source, provide a link to the Creative Commons licence, and indicate if changes were made. The images or other third party material in this article are included in the article's Creative Commons licence, unless indicated otherwise in a credit line to the material. If material is not included in the article's Creative Commons licence and your intended use is not permitted by statutory regulation or exceeds the permitted use, you will need to obtain permission directly from the copyright holder. To view a copy of this licence, visit http://creativecommons.org/licenses/by/4.0/. 
investigated the following generalized family of elliptic integrals (incomplete) $H(\psi, k, \gamma)$ :

$$
\begin{aligned}
& H(\psi, k, \gamma):=\int_{0}^{\psi}\left(1-k^{2} \sin ^{2} \theta\right)^{\gamma-\frac{1}{2}} \mathrm{~d} \theta=\int_{0}^{\sin \psi} \frac{\left(1-k^{2} t^{2}\right)^{\gamma-\frac{1}{2}}}{\sqrt{1-t^{2}}} \mathrm{~d} t \\
& \left(\left|k^{2}\right|<1 ; 0 \leqq \psi \leqq \frac{\pi}{2} ; \gamma \in \mathbb{C}\right) .
\end{aligned}
$$

Obviously, their special cases are

$$
\begin{aligned}
& H\left(\frac{\pi}{2}, k, \gamma\right):=H(k, \gamma), \\
& H(\psi, k, 0)=: F(\psi, k), \quad \text { and } \quad H(\psi, k, 1)=: E(\psi, k),
\end{aligned}
$$

and

$$
H\left(\frac{\pi}{2}, k, 0\right)=: K(k) \text { and } H\left(\frac{\pi}{2}, k, 1\right)=: E(k)
$$

and they respectively yield the families of complete elliptic integrals $H(k, \gamma)$ due to the Bushell [5, p. 2, Eq. (2.2)]

$$
\begin{aligned}
& H(k, \gamma)=\int_{0}^{\frac{\pi}{2}}\left(1-k^{2} \sin ^{2} \theta\right)^{\gamma-\frac{1}{2}} \mathrm{~d} \theta=\int_{0}^{1} \frac{\left(1-k^{2} t^{2}\right)^{\gamma-\frac{1}{2}}}{\sqrt{1-t^{2}}} \mathrm{~d} t \\
& \left(\left|k^{2}\right|<1 ; \gamma \in \mathbb{C}\right)
\end{aligned}
$$

and well-known (canonical) Legendre incomplete elliptic integrals $F(\psi, k)$ and $E(\psi, k)$ and complete elliptic integrals $K(k)$ and $E(k)$ of the first and second kind (in terms of modulus $|k|$ and amplitude $\psi)[6]$ :

$$
\begin{aligned}
& F(\psi, k)=\int_{0}^{\psi} \frac{\mathrm{d} \theta}{\sqrt{1-k^{2} \sin ^{2} \theta}}=\int_{0}^{\sin \psi} \frac{\mathrm{d} t}{\sqrt{\left(1-t^{2}\right)\left(1-k^{2} t^{2}\right)}} \\
& \left(\left|k^{2}\right|<1 ; 0 \leqq \psi \leqq \frac{\pi}{2}\right), \\
& E(\psi, k)=\int_{0}^{\psi} \sqrt{1-k^{2} \sin ^{2} \theta} \mathrm{d} \theta=\int_{0}^{\sin \psi} \sqrt{\frac{1-k^{2} t^{2}}{1-t^{2}}} \mathrm{~d} t \\
& \left(\left|k^{2}\right|<1 ; 0 \leqq \psi \leqq \frac{\pi}{2}\right),
\end{aligned}
$$

and

$$
\begin{aligned}
& K(k)=\int_{0}^{\frac{\pi}{2}} \frac{\mathrm{d} \theta}{\sqrt{1-k^{2} \sin ^{2} \theta}}=\int_{0}^{1} \frac{\mathrm{d} t}{\sqrt{\left(1-t^{2}\right)\left(1-k^{2} t^{2}\right)}} \quad\left(\left|k^{2}\right|<1\right), \\
& E(k)=\int_{0}^{\frac{\pi}{2}} \sqrt{1-k^{2} \sin ^{2} \theta} \mathrm{d} \theta=\int_{0}^{1} \sqrt{\frac{1-k^{2} t^{2}}{1-t^{2}}} \mathrm{~d} t \quad\left(\left|k^{2}\right|<1\right) .
\end{aligned}
$$

In recent years, extensions of a number of well-known special functions have been investigated and studied the $(p, q)$-variant, and in turn, when $p=q$ the $p$-variant together 
with the set of related higher transcendental hypergeometric type special functions (see, for details, [8-10, 17, 18, 20, 22-24]). In what follows we shall use the following recently defined $(p, q)$-extensions of the classical beta function $B(x, y)$ and classical Gauss's hypergeometric function $F(\lambda, \mu ; \nu ; \mathfrak{Z})$ [11, p. 360, Eq. (1.14)]:

$$
\begin{aligned}
& \mathrm{B}_{p, q}(\delta, \sigma)=\mathrm{B}(\delta, \sigma ; p, q)=\int_{0}^{1} t^{\delta-1}(1-t)^{\sigma-1} \mathrm{e}^{-\frac{p}{t}-\frac{q}{1-t}} \mathrm{~d} t \\
& (\min \{\Re(p), \Re(q) ; \min \{\Re(\delta), \Re(\sigma)\}>0\} \geq 0),
\end{aligned}
$$

and [11, p. 371, Eq. (7.1)]

$$
\begin{aligned}
& F_{p, q}(\lambda, \mu ; v ; \mathfrak{Z})=\sum_{n \geq 0}(\lambda)_{n} \frac{\mathrm{B}(\mu+n, v-\mu ; p, q)}{\mathrm{B}(\mu, v-\mu)} \frac{\mathfrak{Z}^{n}}{n !} \\
& (p, q \geq 0 ; \Re(\nu)>\Re(\mu)>0 ;|\mathfrak{Z}|<1) .
\end{aligned}
$$

Also, we shall need a $(p, q)$-extension of Appell's(first) hypergeometric function $F_{1}$ [21, Eq. (1.9)]:

$$
\begin{aligned}
& F_{1}\left(\lambda, \mu, \mu^{\prime} ; v ; \mathfrak{X}, \mathfrak{Y} ; p, q\right)=\sum_{m, n \geq 0}(\mu)_{n}\left(\mu^{\prime}\right)_{n} \frac{\mathrm{B}(\lambda+m+n, v-\lambda ; p, q)}{\mathrm{B}(\lambda, v-\lambda)} \frac{\mathfrak{X}^{m}}{m !} \frac{\mathfrak{Y}^{n}}{n !} \\
& (\min \{\Re(p), \Re(q) ; \max \{|\mathfrak{X}|,|\mathfrak{Y}|\}<1\} \geq 0) .
\end{aligned}
$$

The goal of this paper is to introduce and investigate the family of $(p, q)$-extended (incomplete) elliptic-type integrals and (complete) elliptic-type integrals, which are analogous on the basis of definition (1.7) of the $(p, q)$-extended beta function $\mathrm{B}(\delta, \sigma ; p, q)$ so that many of the known properties of the elliptic-type integrals carry over naturally. In Sect. 2, we introduce a family of $(p, q)$-extended elliptic-type integrals. The $(p, q)$-extension proposed in this section provides connections with $(p, q)$-extended Gauss' hypergeometric function $F_{p, q}$ and $(p, q)$-extended Appell's double hypergeometric function $F_{1}$. In Sect. 3, Turán-type inequalities including the log-convexity property are proved for these $(p, q)$ extended(complete) elliptic-type integrals. Furthermore, in Sect. 4, Mellin transform formulas and some infinite series representations containing the Laguerre polynomials are derived for these $(p, q)$-extended elliptic-type integrals. Moreover, in Sect. 5, we provide certain connections with the $(p, q)$-extended beta function and Meijer's $G$-function of two variables as new representations for the parameter(special) values and differential and integral properties of the $(p, q)$-extended(complete) elliptic-type integrals. Finally, in conclusion Sect. 6, we introduce a $(p, q)$-extension of the Epstein-Hubbell (E-H) elliptic-type integral. 


\section{A family of $(p, q)$-extended elliptic-type integrals}

We begin by introducing a family of $(p, q)$-extended(incomplete) elliptic-type integrals and (complete) elliptic-type integrals (in terms of modulus $|k|$ with amplitude $\psi$ ), given by (1.1)

$$
\begin{aligned}
& \mathcal{H}_{p, q}(\psi, k, \gamma):=\int_{0}^{\psi}\left(1-k^{2} \sin ^{2} \theta\right)^{\gamma-\frac{1}{2}} \mathrm{e}^{-\frac{p}{\sin ^{2} \theta}-\frac{q}{\cos ^{2} \theta}} \mathrm{d} \theta \\
& =\int_{0}^{\sin \psi} \frac{\left(1-k^{2} t^{2}\right)^{\gamma-\frac{1}{2}}}{\sqrt{1-t^{2}}} \mathrm{e}^{-\frac{p}{t^{2}}-\frac{q}{1-t^{2}}} \mathrm{~d} t \\
& \left(\min \{\Re(p), \Re(q)\}>0 ;\left|k^{2}\right|<1 \text { with } 0 \leqq \psi \leqq \frac{\pi}{2} \text { when } p=q=0\right) \text {. }
\end{aligned}
$$

It is clear that from (2.1), in the particular cases $\gamma=0$ and $\gamma=1$, respectively, we have

$$
\begin{aligned}
& F_{p, q}(\psi, k):=\int_{0}^{\psi} \frac{1}{\sqrt{1-k^{2} \sin ^{2} \theta}} \mathrm{e}^{-\frac{p}{\sin ^{2} \theta}-\frac{q}{\cos ^{2} \theta}} \mathrm{d} \theta \\
& =\int_{0}^{\sin \psi} \frac{1}{\sqrt{\left(1-t^{2}\right)\left(1-k^{2} t^{2}\right)}} \mathrm{e}^{-\frac{p}{t^{2}}-\frac{q}{1-t^{2}}} \mathrm{~d} t \\
& \left(\min \{\Re(p), \Re(q)\}>0 ;\left|k^{2}\right|<1 \text { with } 0 \leqq \psi \leqq \frac{\pi}{2} \text { when } p=q=0\right. \text {; ) }
\end{aligned}
$$

and

$$
\begin{aligned}
& E_{p, q}(\psi, k):=\int_{0}^{\psi} \sqrt{1-k^{2} \sin ^{2} \theta} \mathrm{e}^{-\frac{p}{\sin ^{2} \theta}-\frac{q}{\cos ^{2} \theta}} \mathrm{d} \theta \\
& =\int_{0}^{\sin \psi} \sqrt{\frac{1-k^{2} t^{2}}{1-t^{2}}} \mathrm{e}^{-\frac{p}{t^{2}}-\frac{q}{1-t^{2}}} \mathrm{~d} t \\
& \left(\min \{\Re(p), \Re(q)\}>0 ;\left|k^{2}\right|<1 \text { with } 0 \leqq \psi \leqq \frac{\pi}{2} \text { when } p=q=0\right) \text {. }
\end{aligned}
$$

Furthermore, when $\psi=\frac{\pi}{2}$, equations (2.1), (2.2), and (2.3) reduce to the corresponding $(p, q)$-extended(complete) elliptic-type integrals given respectively by

$$
\begin{aligned}
& \mathcal{H}_{p, q}(k, \gamma):=\int_{0}^{\frac{\pi}{2}}\left(1-k^{2} \sin ^{2} \theta\right)^{\gamma-\frac{1}{2}} \mathrm{e}^{-\frac{p}{\sin ^{2} \theta}-\frac{q}{\cos ^{2} \theta}} \mathrm{d} \theta \\
& =\int_{0}^{1} \frac{\left(1-k^{2} t^{2}\right)^{\gamma-\frac{1}{2}}}{\sqrt{1-t^{2}}} \mathrm{e}^{-\frac{p}{t^{2}}-\frac{q}{1-t^{2}}} \mathrm{~d} t, \\
& K_{p, q}(k):=\int_{0}^{\frac{\pi}{2}} \frac{1}{\sqrt{1-k^{2} \sin ^{2} \theta}} \mathrm{e}^{-\frac{p}{\sin ^{2} \theta}-\frac{q}{\cos ^{2} \theta}} \mathrm{d} \theta \\
& =\int_{0}^{1} \frac{1}{\sqrt{\left(1-t^{2}\right)\left(1-k^{2} t^{2}\right)}} \mathrm{e}^{-\frac{p}{t^{2}}-\frac{q}{1-t^{2}}} \mathrm{~d} t \\
& \left(\min \{\Re(p), \Re(q)\}>0 ;\left|k^{2}\right|<1 \text { when } p=q=0\right) \text {, }
\end{aligned}
$$


and

$$
\begin{aligned}
& E_{p, q}(k):=\int_{0}^{\frac{\pi}{2}} \sqrt{1-k^{2} \sin ^{2} \theta} \mathrm{e}^{-\frac{p}{\sin ^{2} \theta}-\frac{q}{\cos ^{2} \theta}} \mathrm{d} \theta \\
& =\int_{0}^{1} \sqrt{\frac{1-k^{2} t^{2}}{1-t^{2}}} \mathrm{e}^{-\frac{p}{t^{2}}-\frac{q}{1-t^{2}}} \mathrm{~d} t \\
& \left(\min \{\Re(p), \Re(q)\}>0 ;\left|k^{2}\right|<1 \text { when } p=q=0\right) \text {. }
\end{aligned}
$$

In terms of the complementary modulus $k^{\prime}$, the $(p, q)$-extended(complete)elliptic-type integrals are defined by

$$
K_{p, q}^{\prime}(k)=K_{p, q}\left(k^{\prime}\right)=K_{p, q}\left(\sqrt{1-k^{2}}\right) \quad\left(k^{\prime}:=\sqrt{1-k^{2}}\right)
$$

and

$$
E_{p, q}^{\prime}(k)=E_{p, q}\left(k^{\prime}\right)=E_{p, q}\left(\sqrt{1-k^{2}}\right) \quad\left(k^{\prime}:=\sqrt{1-k^{2}}\right) .
$$

The particular cases of (2.2) to (2.8) when $p=q$ and $p=q=0$ reduce to the generalized elliptic-type integrals studied by Srivastava et al. [33] and classical elliptic integrals (incomplete and complete) (1.1) to (1.6), respectively (see, e.g., [5, 6], and [12]).

\section{1 $(p, q)$-Hypergeometric(extended) function representations}

In this section, we express the above defined $(p, q)$-extensions of complete elliptic-type integrals in terms of $(p, q)$-extended hypergeometric functions.

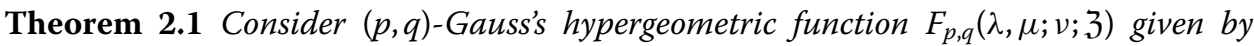
(1.8). Let $\Re(p), \Re(q)>0$, and $\left|k^{2}\right|<1$. Then

$$
\begin{aligned}
& \mathcal{H}_{p, q}(k, \gamma)=\frac{\pi}{2} F_{p, q}\left(\frac{1}{2}-\gamma, \frac{1}{2} ; 1 ; k^{2}\right), \\
& K_{p, q}(k)=\frac{\pi}{2} F_{p, q}\left(\frac{1}{2}, \frac{1}{2} ; 1 ; k^{2}\right),
\end{aligned}
$$

and

$$
E_{p, q}(k)=\frac{\pi}{2} F_{p, q}\left(-\frac{1}{2}, \frac{1}{2} ; 1 ; k^{2}\right) .
$$

Proof Letting $t^{2}=u$ in (2.4), (2.5), and (2.6) and using definition (1.8), we get the required representation (2.9), (2.10), and (2.11), respectively.

Theorem 2.2 Consider $(p, q)$-Appell's hypergeometric function $F_{1}\left(\lambda, \mu, \mu^{\prime} ; \nu ; \mathfrak{X}, \mathfrak{Y} ; p, q\right)$ defined as in (1.9). For $\Re(p), \Re(q)>0$ and $\left|k^{2}\right|<1$, we have

$$
\begin{aligned}
& \mathcal{H}_{p, q}(k, \gamma)=\frac{\pi}{2} F_{1}\left(\frac{1}{2}, \frac{1}{2}, \frac{1}{2}-\gamma ; \frac{3}{2} ; 1, k^{2} ; p, q\right), \\
& K_{p, q}(k)=\frac{\pi}{2} F_{1}\left(\frac{1}{2}, \frac{1}{2}, \frac{1}{2} ; \frac{3}{2} ; 1, k^{2} ; p, q\right),
\end{aligned}
$$




$$
E_{p, q}(k)=\frac{\pi}{2} F_{1}\left(\frac{1}{2}, \frac{1}{2},-\frac{1}{2} ; \frac{3}{2} ; 1, k^{2} ; p, q\right)
$$

Proof Setting $t^{2}=u$ in equations (2.4), (2.5), and (2.6) and using the integral representation defined by Parmar and Pogány [21, Lemma 8, Eqs. (2.1)]

$$
F_{1}\left(\lambda, \mu, \mu^{\prime} ; \nu ; x, y ; p, q\right)=\int_{0}^{1} \frac{t^{\lambda-1}(1-t)^{\nu-\lambda-1}}{\mathrm{~B}(\lambda, v-\lambda)}(1-x t)^{-\mu}(1-y t)^{-\mu^{\prime}} \mathrm{e}^{-\frac{p}{t}-\frac{q}{1-t}} \mathrm{~d} t \text {, }
$$

where $\max \{|\arg (1-\mathfrak{X})|,|\arg (1-\mathfrak{Y})|\}<\pi ; \Re(v)>\Re(\lambda)>0$ and $\min \{\Re(p), \mathfrak{R}(q)\}>0$, we get the required relations (2.12), (2.13), and (2.14), respectively.

\section{Log-convexity properties and Turán-type inequalities}

In this section, we establish the Turán-type inequalities based upon log-convexity properties for the $\mathcal{H}_{p, q}(k, \gamma), K_{p, q}(k)$, and $E_{p, q}(k)$ in (2.4), (2.5), and (2.6).

Theorem 3.1 The following assertions are true for $\Re(p)>0, \Re(q)>0$ :

(1) The function $\gamma \mapsto \mathcal{H}_{p, q}(k, \gamma)$ is log-convex on $(0, \infty)$ for all $k \in(0,1)$.

(2) The function $p \mapsto \mathcal{H}_{p, q}(k, \gamma)$ is log-convex on $(0, \infty)$ for all $k \in(0,1)$.

(3) The function $q \mapsto \mathcal{H}_{p, q}(k, \gamma)$ is log-convex on $(0, \infty)$ for all $k \in(0,1)$.

Moreover, for the same parametric range, the following Turán inequalities hold true:

$$
\begin{aligned}
& \mathcal{H}_{p, q}^{2}(k, \gamma)-\mathcal{H}_{p, q}(k, \gamma-1) \cdot \mathcal{H}_{p, q}(k, \gamma+1) \leq 0 \quad \gamma \in(1, \infty), \\
& \mathcal{H}_{p, q}^{2}(k, \gamma)-\mathcal{H}_{p-1, q}(k, \gamma) \cdot \mathcal{H}_{p+1, q}(k, \gamma) \leq 0 \quad p \in(1, \infty), \\
& \mathcal{H}_{p, q}^{2}(k, \gamma)-\mathcal{H}_{p, q-1}(k, \gamma) \cdot \mathcal{H}_{p, q+1}(k, \gamma) \leq 0 \quad q \in(1, \infty)
\end{aligned}
$$

Proof By using the definition of the classical Hölder-Rogers inequality for integrals in the integral representation (2.4), we have

$$
\begin{aligned}
\mathcal{H}_{p, q} & \left(k, \lambda \gamma_{1}+(1-\lambda) \gamma_{2}\right) \\
& =\int_{0}^{1} \frac{\left(1-k^{2} t^{2}\right)^{\lambda \gamma_{1}+(1-\lambda) \gamma_{2}-\frac{1}{2}}}{\sqrt{1-t^{2}}} \mathrm{e}^{-\frac{p}{t^{2}}-\frac{q}{1-t^{2}}} \mathrm{~d} t \\
& =\int_{0}^{1}\left[\frac{\left(1-k^{2} t^{2}\right)^{\gamma_{1}-\frac{1}{2}}}{\sqrt{1-t^{2}}} \mathrm{e}^{-\frac{p}{t^{2}}-\frac{q}{1-t^{2}}}\right]^{\lambda}\left[\frac{\left(1-k^{2} t^{2}\right)^{\gamma_{2}-\frac{1}{2}}}{\sqrt{1-t^{2}}} \mathrm{e}^{-\frac{p}{t^{2}}-\frac{q}{1-t^{2}}}\right]^{1-\lambda} \mathrm{d} t \\
& \leq\left[\int_{0}^{1} \frac{\left(1-k^{2} t^{2}\right)^{\gamma_{1}-\frac{1}{2}}}{\sqrt{1-t^{2}}} \mathrm{e}^{-\frac{p}{t^{2}}-\frac{q}{1-t^{2}}} \mathrm{~d} t\right]^{\lambda}\left[\int_{0}^{1} \frac{\left(1-k^{2} t^{2}\right)^{\gamma_{2}-\frac{1}{2}}}{\sqrt{1-t^{2}}} \mathrm{e}^{-\frac{p}{t^{2}}-\frac{q}{1-t^{2}}} \mathrm{~d} t\right]^{1-\lambda} .
\end{aligned}
$$

This is equivalent to

$$
\begin{aligned}
& \mathcal{H}_{p, q}\left(k, \lambda \gamma_{1}+(1-\lambda) \gamma_{2}\right) \leq\left[\mathcal{H}_{p, q}\left(k, \gamma_{1}\right)\right]^{\lambda}\left[\mathcal{H}_{p, q}\left(k, \gamma_{2}\right)\right]^{1-\lambda} \\
& \forall \gamma_{1}, \gamma_{2}, k \in(0,1) \text { and } \lambda \in[0,1]
\end{aligned}
$$

which proves the first assertion.

In a similar manner, by using (2.4) and using the Hölder-Rogers inequality, we get

$$
\begin{aligned}
& \mathcal{H}_{\lambda p_{1}+(1-\lambda) p_{2}, q}(k, \gamma) \leq\left[\mathcal{H}_{p_{1}, q}(k, \gamma)\right]^{\lambda}\left[\mathcal{H}_{p_{2}, q}(k, \gamma)\right]^{1-\lambda} \\
& \forall p_{1}, p_{2}>0, k \in(0,1) \text { and } \lambda \in[0,1]
\end{aligned}
$$




$$
\begin{aligned}
& \mathcal{H}_{p, \lambda q_{1}+(1-\lambda) q_{2}}(k, \gamma) \leq\left[\mathcal{H}_{p, q_{1}}(k, \gamma)\right]^{\lambda}\left[\mathcal{H}_{p, q_{2}}(k, \gamma)\right]^{1-\lambda} \\
& \forall q_{1}, q_{2}>0, k \in(0,1) \text { and } \lambda \in[0,1]
\end{aligned}
$$

which proves the second assertion and the third assertion.

Next, choosing $\lambda=\frac{1}{2}$ in (3.4), (3.5), and (3.6), we conclude the Turán inequalities (3.1), (3.2), and (3.3), respectively.

Corollary 3.1 The following assertions are true for $\Re(p)>0, \Re(q)>0$ :

(1) The function $p \mapsto K_{p, q}(k)$ is log-convex on $(0, \infty)$ for all $k \in(0,1)$.

(2) The function $q \mapsto K_{p, q}(k)$ is log-convex on $(0, \infty)$ for all $k \in(0,1)$.

(3) The function $p \mapsto E_{p, q}(k)$ is log-convex on $(0, \infty)$ for all $k \in(0,1)$.

(4) The function $q \mapsto E_{p, q}(k)$ is log-convex on $(0, \infty)$ for all $k \in(0,1)$.

Moreover, for the same parametric range, the following Turán inequalities hold true:

$$
\begin{array}{ll}
K_{p, q}^{2}(k)-K_{p-1, q}(k) \cdot K_{p+1, q}(k) \leq 0 & p \in(1, \infty), \\
K_{p, q}^{2}(k)-K_{p, q-1}(k) \cdot K_{p, q+1}(k) \leq 0 & q \in(1, \infty), \\
E_{p, q}^{2}(k)-E_{p-1, q}(k) \cdot E_{p+1, q}(k) \leq 0 & p \in(1, \infty), \\
E_{p, q}^{2}(k)-E_{p, q-1}(k) \cdot E_{p, q+1}(k) \leq 0 & q \in(1, \infty) .
\end{array}
$$

Proof Furthermore, by specifying $\gamma=0$ and $\gamma=1$ in assertions (3.5) and (3.6) (or alternatively employing definitions (2.5) and (2.6) and using the Hölder-Rogers inequality), we can easily obtain

$$
\begin{array}{ll}
K_{\lambda p_{1}+(1-\lambda) p_{2}, q}(k) \leq\left[K_{p_{1}, q}(r)\right]^{\lambda}\left[K_{p_{2}, q}(r)\right]^{1-\lambda} \quad \forall p_{1}, p_{2}>0, k \in(0,1) \text { and } \lambda \in[0,1], \\
K_{p, \lambda q_{1}+(1-\lambda) q_{2}}(k) \leq\left[K_{p, q_{1}}(r)\right]^{\lambda}\left[K_{p, q_{2}}(r)\right]^{1-\lambda} \quad \forall q_{1}, q_{2}>0, k \in(0,1) \text { and } \lambda \in[0,1], \\
E_{\lambda p_{1}+(1-\lambda) p_{2}, q}(k) \leq\left[E_{p_{1}, q}(r)\right]^{\lambda}\left[E_{p_{2}, q}(r)\right]^{1-\lambda} \quad \forall p_{1}, p_{2}>0, k \in(0,1) \text { and } \lambda \in[0,1]
\end{array}
$$

and

$$
E_{p, \lambda q_{1}+(1-\lambda) q_{2}}(k) \leq\left[E_{p, q_{1}}(r)\right]^{\lambda}\left[E_{p, q_{2}}(r)\right]^{1-\lambda} \quad \forall q_{1}, q_{2}>0, k \in(0,1) \text { and } \lambda \in[0,1]
$$

Again, specifying $\lambda=\frac{1}{2}$ and $p_{1}=p-1, p_{2}=p+1$ in (3.11) and (3.13) and $q_{1}=q-1, q_{2}=q+1$ in (3.12) and (3.14), we obtain the Turán-type inequalities (3.7) to (3.10), respectively.

\section{Mellin transform formulas and Laguerre polynomial representations}

The Mellin transforms of the function $f(x, y)$ of two variables with respect to the indices $r$ and $s$ are given by [19]

$$
\mathcal{M}\{f(x, y)\}(r, s)=\int_{0}^{\infty} \int_{0}^{\infty} x^{r-1} y^{s-1} f(x, y) \mathrm{d} x \mathrm{~d} y
$$

where it is assumed that the integral (improper) in (4.1) exists. 
Theorem 4.1 Consider Appell's hypergeometric function $F_{1}\left(\lambda, \mu, \mu^{\prime} ; \nu ; \mathfrak{X}, \mathfrak{Y}\right)$. The following Mellin transformation formula for $\mathcal{H}_{p, q}(\psi, k, \gamma)$ in (2.1) holds true:

$$
\begin{aligned}
& \mathcal{M}\left\{\mathcal{H}_{p, q}(\psi, k, \gamma)\right\}(r, s)= \frac{\Gamma(r) \Gamma(s) \sin ^{2 r+1} \psi}{2\left(r+\frac{1}{2}\right)} \\
& \cdot F_{1}\left(r+\frac{1}{2}, \frac{1}{2}-\gamma, \frac{1}{2}-s ; r+\frac{3}{2} ; k^{2} \sin ^{2} \psi, \sin ^{2} \psi\right) \\
&\left(\Re(r)>0, \Re(s)>0 ;\left|k^{2}\right|<1 ; 0 \leqq \psi \leqq \frac{\pi}{2}\right) .
\end{aligned}
$$

Proof By making use of definition (4.1), we get from (2.1) that

$$
\begin{aligned}
\mathcal{M} & \left\{\mathcal{H}_{p, q}(\psi, k, \gamma)\right\}(r, s) \\
& =\int_{0}^{\infty} \int_{0}^{\infty} p^{r-1} q^{s-1} \mathcal{H}_{p, q}(\psi, k, \gamma) \mathrm{d} p \mathrm{~d} q \\
& =\int_{0}^{\infty} \int_{0}^{\infty} p^{r-1} q^{s-1}\left(\int_{0}^{\psi}\left(1-k^{2} \sin ^{2} \theta\right)^{\gamma-\frac{1}{2}} \mathrm{e}^{-\frac{p}{\sin ^{2} \theta}-\frac{q}{\cos ^{2} \theta}} \mathrm{d} \theta\right) \mathrm{d} p \mathrm{~d} q \\
& =\frac{1}{2} \int_{0}^{\infty} \int_{0}^{\infty} p^{r-1} q^{s-1}\left(\int_{0}^{\sin ^{2} \psi} \frac{\left(1-k^{2} t\right)^{\gamma-\frac{1}{2}}}{\sqrt{t(1-t)}} \mathrm{e}^{-\frac{p}{t}-\frac{q}{1-t}} \mathrm{~d} t\right) \mathrm{d} p \mathrm{~d} q,
\end{aligned}
$$

where the value in the inner $\theta$-integral has also been evaluated by $\sin ^{2} \theta=t$ and $\mathrm{d} \theta=$ $\frac{\mathrm{d} t}{2 \sqrt{t(1-t)}}$. Now, by the absolute convergence of the integrations involved under the conditions mentioned in Theorem 4.1, we change the order of integrals on the right-hand side (RHS) to find

$$
\begin{aligned}
\mathcal{M} & \left\{\mathcal{H}_{p, q}(\psi, k, \gamma)\right\}(r, s) \\
= & \frac{1}{2} \int_{0}^{\sin ^{2} \psi} \frac{\left(1-k^{2} t\right)^{\gamma-\frac{1}{2}}}{\sqrt{t(1-t)}}\left(\int_{0}^{\infty} p^{r-1} \mathrm{e}^{-\frac{p}{t}} \mathrm{~d} p\right)\left(\int_{0}^{\infty} q^{s-1} \mathrm{e}^{-\frac{q}{1-t}} \mathrm{~d} q\right) \mathrm{d} t \\
= & \frac{\Gamma(r) \Gamma(s)}{2} \int_{0}^{\sin ^{2} \psi} t^{r-\frac{1}{2}}(1-t)^{s-\frac{1}{2}}\left(1-k^{2} t\right)^{\gamma-\frac{1}{2}} \mathrm{~d} t,
\end{aligned}
$$

where we obviously have used the classical Euler's gamma integral

$$
\Gamma(\eta) \xi^{-\eta}=\int_{0}^{\infty} e^{-\xi t} t^{\eta-1} \mathrm{~d} t, \quad \Re(\xi)>0, \Re(\eta)>0,
$$

in the inner $p$-integral and $q$-integral. Now, setting $t=\left(\sin ^{2} \psi\right) \tau$ and $\mathrm{d} t=\left(\sin ^{2} \psi\right) \mathrm{d} \tau$ yields

$$
\begin{aligned}
& \mathcal{M}\left\{\mathcal{H}_{p, q}(\psi, k, \gamma)\right\}(r, s) \\
& =\frac{\Gamma(r) \Gamma(s) \sin ^{2 r+1} \psi}{2} \int_{0}^{1} \tau^{r-\frac{1}{2}}\left(1-k^{2} \sin ^{2} \psi \tau\right)^{\gamma-\frac{1}{2}}\left(1-\sin ^{2} \psi \tau\right)^{s-\frac{1}{2}} \mathrm{~d} \tau .
\end{aligned}
$$


Finally, by using (see, e.g., [32, p. 276, Eq. 9.4(7)])

$$
\begin{aligned}
& F_{1}\left(\lambda, \mu, \mu^{\prime} ; \nu ; \mathfrak{X}, \mathfrak{Y}\right)=\frac{\Gamma(\nu)}{\Gamma(\lambda) \Gamma(v-\lambda)} \int_{0}^{1} t^{\lambda-1}(1-t)^{\nu-\lambda-1}(1-\mathfrak{X} t)^{-\mu}(1-\mathfrak{Y} t)^{-\mu^{\prime}} \mathrm{d} t \\
& (\max \{|\arg (1-\mathfrak{X})|,|\arg (1-\mathfrak{Y})|\}<\pi ; \Re(\nu)>\Re(\lambda)>0),
\end{aligned}
$$

we obtain the required Mellin transformation formula (4.2) given by Theorem 4.1.

Appell's functions $F_{1}$ are represented in terms of Kampé de Fériet's hypergeometric function [32, p. 22, Eq. 1.3(2)] and the Srivastava-Daoust hypergeometric function of the two variables [32, p. 37, Eq. 1.4(21)]:

$$
F_{1}\left[\alpha, \beta_{1}, \beta_{2} ; \gamma ; \mathfrak{X}, \mathfrak{Y}\right]=F_{1: 0 ; 0}^{1: 1 ; 1}\left[\begin{array}{l}
\alpha: \beta_{1} ; \beta_{2} ; \mathfrak{X}, \mathfrak{Y} \\
\gamma:-;-;
\end{array}\right]
$$

and

$$
F_{1}\left[\alpha, \beta_{1}, \beta_{2} ; \gamma ; \mathfrak{X}, \mathfrak{Y}\right]=F_{1: 0 ; 0}^{1: 1 ; 1}\left[\begin{array}{c}
\left.(\alpha: 1,1):\left(\beta_{1}, 1\right) ;\left(\beta_{2}, 1\right) ; \mathfrak{X}, \mathfrak{Y}\right) \\
(\gamma: 1,1): \longleftarrow
\end{array}\right.
$$

We first apply representation (4.7) in (4.2) and representation (4.8) in (4.2), and afterward employing the Legendre duplication formula [32, p. 17, Eq. 1.2(14)]) for the gamma function

$$
\Gamma(2 \eta)=\frac{2^{2 \eta-1}}{\sqrt{\pi}} \Gamma(\eta) \Gamma\left(\eta+\frac{1}{2}\right)
$$

we can evaluate the Mellin transform formulas for $\mathcal{H}_{p, q}(\psi, k, \gamma)$ in (2.1) as given by Corollary 4.1. In particular, if we put $\gamma=0$ and $\gamma=1$, we can obtain certain Mellin transformation formulas for the $F_{p, q}(\psi, k)$ and $E_{p, q}(\psi, k)$ in (2.2) and (2.3) as given by Corollary 4.2. The proofs are omitted for Corollaries 4.1 and 4.2 .

Corollary 4.1 The following Mellin transformation formulas hold true:

$$
\begin{aligned}
\mathcal{M}\left\{\mathcal{H}_{p, q}(\psi, k, \gamma)\right\}(r, s)= & \frac{\Gamma(r) \Gamma(s) \sin ^{2 r+1} \psi}{2\left(r+\frac{1}{2}\right)} \\
& \cdot F_{1: 0 ; 0}^{1: 1 ; 1}\left[\begin{array}{c}
r+\frac{1}{2}: \frac{1}{2}-\gamma ; \frac{1}{2}-s ; k^{2} \sin ^{2} \psi, \sin ^{2} \psi \\
r+\frac{3}{2}:-;
\end{array}\right]
\end{aligned}
$$

and

$$
\begin{aligned}
& \mathcal{M}\left\{\mathcal{H}_{p, q}(\psi, k, \gamma)\right\}(r, s) \\
& =\Gamma(r) \Gamma(s) \sin ^{2 r+1} \psi \\
& \cdot F_{1: 0 ; 0}^{1: 1 ; 1}\left[\begin{array}{c}
(2 r+1: 2,2):\left(\frac{1}{2}-\gamma, 1\right) ;\left(\frac{1}{2}-s, 1\right) ; \\
\left.(2 r+2: 2,2):-; k^{2} \sin ^{2} \psi, \sin ^{2} \psi\right] .
\end{array}\right.
\end{aligned}
$$


Corollary 4.2 The following Mellin transformation formulas for $F_{p, q}(\psi, k)$ and $E_{p, q}(\psi, k)$ in (2.2) and (2.3) hold true:

$$
\begin{aligned}
& \mathcal{M}\left\{F_{p, q}(\psi, k)\right\}(r, s)=\frac{\Gamma(r) \Gamma(s) \sin ^{2 r+1} \psi}{2\left(r+\frac{1}{2}\right)} \\
& \cdot F_{1}\left(r+\frac{1}{2}, \frac{1}{2}, \frac{1}{2}-s ; r+\frac{3}{2} ; k^{2} \sin ^{2} \psi, \sin ^{2} \psi\right), \\
& \mathcal{M}\left\{F_{p, q}(\psi, k)\right\}(r, s)=\frac{\Gamma(r) \Gamma(s) \sin ^{2 r+1} \psi}{2\left(r+\frac{1}{2}\right)} \\
& \times F_{1: 0 ; 0}^{1: 1 ; 1}\left[\begin{array}{c}
r+\frac{1}{2}: \frac{1}{2} ; \frac{1}{2}-s ; \\
r+\frac{3}{2}:-; k^{2} \sin ^{2} \psi, \sin ^{2} \psi
\end{array} ;\right. \text {, } \\
& \mathcal{M}\left\{F_{p, q}(\psi, k)\right\}(r, s) \\
& =\Gamma(r) \Gamma(s) \sin ^{2 r+1} \psi \\
& \cdot F_{1: 0 ; 0}^{1: 1 ; 1}\left[\begin{array}{c}
(2 r+1: 2,2):\left(\frac{1}{2}, 1\right) ;\left(\frac{1}{2}-s, 1\right) ; \\
(2 r+2: 2,2): \square
\end{array} ; k^{2} \sin ^{2} \psi, \sin ^{2} \psi\right], \\
& \mathcal{M}\left\{E_{p, q}(\psi, k)\right\}(r, s) \\
& =\frac{\Gamma(r) \Gamma(s) \sin ^{2 r+1} \psi}{2\left(r+\frac{1}{2}\right)} \cdot F_{1}\left(r+\frac{1}{2},-\frac{1}{2}, \frac{1}{2}-s ; r+\frac{3}{2} ; k^{2} \sin ^{2} \psi, \sin ^{2} \psi\right), \\
& \mathcal{M}\left\{E_{p, q}(\psi, k)\right\}(r, s) \\
& =\frac{\Gamma(r) \Gamma(s) \sin ^{2 r+1} \psi}{2\left(r+\frac{1}{2}\right)} F_{1: 0 ; 0}^{1: 1 ; 1}\left[\begin{array}{c}
r+\frac{1}{2}:-\frac{1}{2} ; \frac{1}{2}-s ; \\
r+\frac{3}{2}: \square
\end{array} k^{2} \sin ^{2} \psi, \sin ^{2} \psi\right],
\end{aligned}
$$

and

$$
\begin{aligned}
\mathcal{M} & \left\{E_{p, q}(\psi, k)\right\}(r, s) \\
= & \Gamma(r) \Gamma(s) \sin ^{2 r+1} \psi \\
& \times F_{1: 0 ; 0}^{1: 1 ; 1}\left[\begin{array}{r}
(2 r+1: 2,2):\left(-\frac{1}{2}, 1\right) ;\left(\frac{1}{2}-s, 1\right) ; \\
(2 r+2: 2,2):
\end{array} ; k^{2} \sin ^{2} \psi, \sin ^{2} \psi\right] .
\end{aligned}
$$

Theorem 4.2 The mentioned Mellin transformation formulas for $\mathcal{H}_{p, q}(k, \gamma), K_{p, q}(k)$, and $E_{p, q}(k)$ in (2.4), (2.5), and (2.6) hold true:

$$
\begin{aligned}
& \mathcal{M}\left\{\mathcal{H}_{p, q}(k, \gamma)\right\}(r, s)=\frac{\Gamma(r) \Gamma(s) B\left(r+\frac{1}{2}, s+\frac{1}{2}\right)}{2}{ }_{2} F_{1}\left(r+\frac{1}{2}, \frac{1}{2}-\gamma ; r+s+1 ; k^{2}\right) \\
& \left(\Re(r)>0, \Re(s)>0 ;\left|k^{2}\right|<1 ; 0 \leqq \psi \leqq \frac{\pi}{2}\right), \\
& \mathcal{M}\left\{K_{p, q}(k)\right\}(r, s)=\frac{\Gamma(r) \Gamma(s) B\left(r+\frac{1}{2}, s+\frac{1}{2}\right)}{2}{ }_{2} F_{1}\left(r+\frac{1}{2}, \frac{1}{2} ; r+s+1 ; k^{2}\right) \\
& \left(\Re(r)>0, \Re(s)>0 ;\left|k^{2}\right|<1 ; 0 \leqq \psi \leqq \frac{\pi}{2}\right),
\end{aligned}
$$


and

$$
\begin{aligned}
& \mathcal{M}\left\{E_{p, q}(k)\right\}(r, s)=\frac{\Gamma(r) \Gamma(s) B\left(r+\frac{1}{2}, s+\frac{1}{2}\right)}{2}{ }_{2} F_{1}\left(r+\frac{1}{2},-\frac{1}{2} ; r+s+1 ; k^{2}\right) \\
& \left(\Re(s)>0 ;\left|k^{2}\right|<1 ; 0 \leqq \psi \leqq \frac{\pi}{2}\right) .
\end{aligned}
$$

Proof By first substituting $\psi=\frac{\pi}{2}$ in (4.2), (4.11), and (4.14) and afterward employing the following identity (see [13, p. 239, Eq. (10)])

$$
F_{1}\left[\lambda, \mu_{1}, \mu_{2} ; v ; \mathfrak{X}, 1\right]=\frac{\Gamma(v) \Gamma\left(v-\lambda-\mu_{2}\right)}{\Gamma(v-\lambda) \Gamma\left(v-\mu_{2}\right)}{ }_{2} F_{1}\left(\lambda, \mu_{1} ; v-\mu_{2} ; \mathfrak{X}\right),
$$

we obtain the required Mellin transformation formulas given by Theorem 4.2.

If we set $r=1$ and $s=1$ in (4.18) and (4.19), we obtain the following connection between the classical elliptic integrals and the $(p, q)$-extended (complete) elliptic integrals:

$$
\int_{0}^{\infty} \int_{0}^{\infty} K_{p, q}(k) \mathrm{d} p \mathrm{~d} q=\frac{\pi}{16}{ }_{2} F_{1}\left(\frac{1}{2}, \frac{3}{2} ; 3 ; k^{2}\right)
$$

which, in view of the well-known representation [26, p. 473, Entry (93)], yields

$$
\int_{0}^{\infty} \int_{0}^{\infty} K_{p, q}(k) \mathrm{d} p \mathrm{~d} q=\frac{1}{3 k^{2}}\left[K(k)-\left(2-k^{2}\right) D(k)\right]
$$

and

$$
\int_{0}^{\infty} \int_{0}^{\infty} E_{p, q}(k) \mathrm{d} p \mathrm{~d} q=\frac{\pi}{16}{ }_{2} F_{1}\left(-\frac{1}{2}, \frac{3}{2} ; 3 ; k^{2}\right)
$$

which, by means of the well-known representation [26, p. 469, Entry (20)], can be written in the following form:

$$
\int_{0}^{\infty} \int_{0}^{\infty} E_{p, q}(k) \mathrm{d} p \mathrm{~d} q=\frac{1}{15 k^{2}}\left[\left(1+k^{2}\right) K(k)-2\left(1-k^{2}+k^{4}\right) D(k)\right]
$$

The Laguerre(simple)polynomials $L_{n}(x)$ are defined by (see, e.g., [25, p. 645])

$$
L_{n}(x):=L_{n}^{(0)}(x) \quad \text { and } \quad L_{n}^{(\lambda)}(x):=\sum_{j=0}^{n}\left(\begin{array}{c}
n+\lambda \\
n-j
\end{array}\right) \frac{(-x)^{j}}{j !}
$$

Now we derive the Laguerre polynomial relation asserted by (4.24).

Theorem 4.3 The following Laguerre polynomial relation holds true for $\mathcal{H}_{p, q}(k)$ :

$$
\mathcal{H}_{p, q}(k)=\sum_{m, n=0}^{\infty} \frac{e^{-p-q} B\left(n+\frac{3}{2}, m+\frac{3}{2}\right)}{2} L_{m}(p) L_{n}(q)_{2} F_{1}\left(\gamma-\frac{1}{2}, n+\frac{3}{2} ; m+n+3 ; k^{2}\right) .
$$


Proof Putting $\sin ^{2} \theta=t$ and $2 \mathrm{~d} \theta=\mathrm{d} t / \sqrt{t(1-t)}$ in (2.4), we get

$$
\mathcal{H}_{p, q}(k)=\frac{1}{2} \int_{0}^{1} t^{-\frac{1}{2}}(1-t)^{-\frac{1}{2}}\left(1-k^{2} t\right)^{\gamma-\frac{1}{2}} \mathrm{e}^{-\frac{p}{t}-\frac{q}{1-t}} \mathrm{~d} t
$$

By making use of the known identity in a slightly corrected form for the simple Laguerre polynomials due to Choi et al. [11, p. 350, Eq. (5.5)]

$$
\exp \left(-\frac{p}{t}-\frac{q}{1-t}\right)=e^{-p-q}\left\{\sum_{m, n=0}^{\infty} L_{m}(p) L_{n}(q) t^{n+1}(1-t)^{m+1}\right\}
$$

in (4.25), we have

$$
\begin{aligned}
& \mathcal{H}_{p, q}(k) \\
& \quad=\frac{e^{-p-q}}{2} \int_{0}^{1} t^{-\frac{1}{2}}(1-t)^{-\frac{1}{2}}\left(1-k^{2} t\right)^{\gamma-\frac{1}{2}}\left\{\sum_{m, n=0}^{\infty} L_{m}(p) L_{n}(q) t^{n+1}(1-t)^{m+1}\right\} d t .
\end{aligned}
$$

Now, changing integration and summation order and using the integral representation of ${ }_{2} F_{1}[27]$

$$
{ }_{2} F_{1}(\lambda, \mu ; \nu ; \mathfrak{Z})=\frac{\Gamma(\nu)}{\Gamma(\mu) \Gamma(v-\mu)} \int_{0}^{1} t^{\mu-1}(1-t)^{\nu-\mu-1}(1-\mathfrak{Z} t)^{-\lambda} \mathrm{d} t,
$$

when there holds $\mathfrak{R}(\nu)>\mathfrak{R}(\mu)>0 ;|\arg (1-\mathfrak{Z})| \leq \pi-\epsilon(0<\epsilon<\pi)$ in (4.26), we are led to the required result.

Corollary 4.3 The following Laguerre polynomial relations hold true for $K_{p, q}(k)$ and $E_{p, q}(k)$ :

$$
K_{p, q}(k)=\sum_{m, n=0}^{\infty} \frac{e^{-p-q} B\left(n+\frac{3}{2}, m+\frac{3}{2}\right)}{2} L_{m}(p) L_{n}(q){ }_{2} F_{1}\left(\frac{1}{2}, n+\frac{3}{2} ; m+n+3 ; k^{2}\right)
$$

and

$$
E_{p, q}(k)=\sum_{m, n=0}^{\infty} \frac{e^{-p-q} B\left(n+\frac{3}{2}, m+\frac{3}{2}\right)}{2} L_{m}(p) L_{n}(q){ }_{2} F_{1}\left(-\frac{1}{2}, n+\frac{3}{2} ; m+n+3 ; k^{2}\right) .
$$

\section{Certain properties of $(p, q)$-extended elliptic integrals}

In this section we obtain certain special values in terms of $(p, q)$-extended beta function $\mathrm{B}_{p, q}(\delta, \sigma)$ and present the connections with $G$-function of two variables. We also present various derivative and integrals formulas for the $(p, q)$-extended elliptic-type integrals.

\subsection{Special parametric values and connections with $G$-function}

In this subsection, we first find the special values of $K_{p, q}(k), K_{p, q}^{\prime}(k), E_{p, q}(k)$, and $E_{p, q}^{\prime}(k)$ in terms of the $(p, q)$-extended beta function $\mathrm{B}_{p, q}(\delta, \sigma)$. It suffices to consider the corresponding defining expressions in Sect. 2 in view of the definition of $\mathrm{B}_{p, q}(\delta, \sigma)(1.7)$. 
Theorem 5.1 Let $\mathrm{B}_{p, q}(\delta, \sigma)$ be the $(p, q)$-extended beta function defined in (1.7). Then:

$$
\begin{aligned}
& K_{p, q}(0)=K_{p, q}^{\prime}(1)=E_{p, q}(0)=E_{p, q}^{\prime}(1)=\frac{1}{2} \mathrm{~B}_{p, q}\left(\frac{1}{2}, \frac{1}{2}\right), \\
& E_{p, q}(1)=E_{p, q}^{\prime}(0)=\frac{1}{2} \mathrm{~B}_{p, q}\left(\frac{1}{2}, 1\right),
\end{aligned}
$$

and

$$
K_{p, q}(1)=K_{p, q}^{\prime}(0)=\frac{1}{2} \mathrm{~B}_{p, q}\left(\frac{1}{2}, 0\right)
$$

Next, observe that Meijer's G-function of two variables [31, p.7, Eq. (1.2.3) and p.88, Eq. (6.4.1)] is expressible in terms of the $(p, q)$-extended beta function $\mathrm{B}_{p, q}(\delta, \sigma)$ for $\Re(p), \Re(q)>0$ as follows [11, p.350, Eq. (5.6)]:

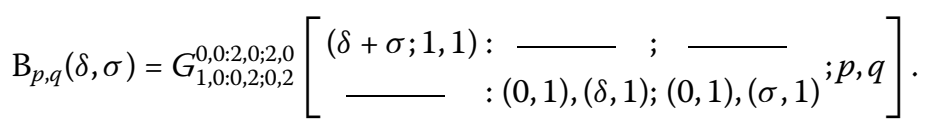

Now, by making use of relationship (5.4) to (5.1) through (5.3) in Theorem 5.1, we can establish various representations of $(p, q)$-extended (complete) elliptic integrals that are given in Corollary 5.1.

Corollary 5.1 The following relations hold true:

$$
\begin{aligned}
& K_{p, q}(0)=K_{p, q}^{\prime}(1)=E_{p, q}(0)=E_{p, q}^{\prime}(1)
\end{aligned}
$$

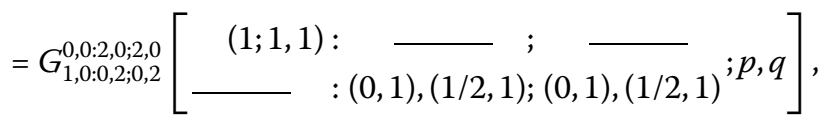

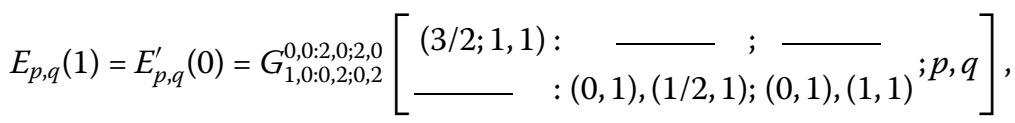

and

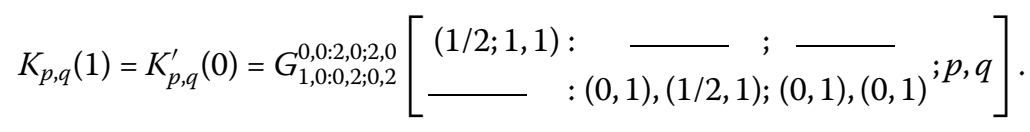

\subsection{Differential and integral formulas}

In this subsection, we present various differential and integral formulas for $(p, q)$-extended elliptic-type integrals. The proofs are omitted.

Theorem 5.2 The following derivative formulas hold true for $K_{p, q}(k)$ and $E_{p, q}(k)$ :

$$
\begin{aligned}
& \frac{\mathrm{d}}{\mathrm{d} k}\left\{E_{p, q}(\psi, k)\right\}=\frac{1}{k}\left[E_{p, q}(\psi, k)-F_{p, q}(\psi, k)\right], \\
& \frac{\mathrm{d}}{\mathrm{d} k}\left\{E_{p, q}(k)\right\}=\frac{1}{k}\left[E_{p, q}(k)-K_{p, q}(k)\right], \\
& \frac{\mathrm{d}}{\mathrm{d} k}\left\{E_{p, q}^{\prime}(k)\right\}=-\frac{k}{k^{\prime 2}}\left[E_{p, q}^{\prime}(k)-K_{p, q}^{\prime}(k)\right],
\end{aligned}
$$




$$
\begin{aligned}
& \frac{\mathrm{d}}{d k^{\prime}}\left\{E_{p, q}(k)\right\}=-\frac{k^{\prime}}{k^{2}}\left[E_{p, q}(k)-K_{p, q}(k)\right], \\
& \frac{\mathrm{d}^{m}}{d\left(k^{2}\right)^{m}}\left\{K_{p, q}(k)\right\}=\frac{\pi[(2 m) !]^{2}}{2(m !)^{3} 16^{m}} F_{p, q}\left(\frac{1}{2}+m, \frac{1}{2}+m, 1+m ; k^{2}\right), \\
& \frac{\mathrm{d}^{m}}{d\left(k^{2}\right)^{m}}\left\{E_{p, q}(k)\right\}=\frac{\pi}{2} \frac{\left(\frac{1}{2}\right)_{m}\left(-\frac{1}{2}\right)_{m}}{m !} F_{p, q}\left(-\frac{1}{2}+m, \frac{1}{2}+m, 1+m ; k^{2}\right) .
\end{aligned}
$$

Theorem 5.3 The following integral formulas hold true for $K_{p, q}(k)$ and $E_{p, q}(k)$ :

$$
\begin{aligned}
& \int\left[K_{p, q}(k)-E_{p, q}(k)\right] \frac{\mathrm{d} k}{k}=-E_{p, q}(k), \\
& \int K_{p, q}(k) \frac{\mathrm{d} k}{k^{2}}=-\frac{1}{k} E_{p, q}(k), \\
& \int \frac{k}{k^{\prime 2}}\left[K_{p, q}^{\prime}(k)-E_{p, q}^{\prime}(k)\right] \mathrm{d} k=E_{p, q}^{\prime}(k),
\end{aligned}
$$

and

$$
\int \frac{k^{\prime}}{k^{2}}\left[K_{p, q}(k)-E_{p, q}^{\prime}(k)\right] \mathrm{d} k^{\prime}=E_{p, q}(k)
$$

\section{Concluding remark and observations}

In our present studies, we have introduced and extensively investigated the family of (incomplete and complete) $(p, q)$-extended elliptic-type integrals and presented connections with $(p, q)$-extended beta function, $(p, q)$-extended Gauss' hypergeometric function, and $(p, q)$-extended Appell's double hypergeometric function $F_{1}$. Moreover, we obtained the connection with Meijer $G$-function of two variables. Turán-type inequalities were proved by using log-convexity property for these $(p, q)$-extended complete elliptic-type integrals. Further, we established various Mellin transform formulas and obtained certain infinite series representations containing Laguerre polynomials. We also obtained some relationship between these $(p, q)$-extended elliptic-type integrals and as special values and deduced numerous differentiation and integral formulas. In conclusion, we introduced $(p, q)$ extension of the Epstein-Hubbell elliptic-type integral.

It is worth mentioning, as a main conclusion to observe, that Epstein and Hubbell [12] studied and investigated the following extensions of $K(k)$ and $E(k)$, which was encountered in a Legendre polynomial expansion method when applied to certain problems involving computation of the radiation field off-axis from a uniform circular disk radiating according to an arbitrary angular distribution law [14] (see also Weiss [37]):

$$
\begin{aligned}
& \Omega_{j}(\kappa):=\int_{0}^{\pi} \frac{\mathrm{d} \theta}{\left(1-\kappa^{2} \cos \theta\right)^{j+\frac{1}{2}}} \\
& \left(0 \leqq \kappa<1 ; j \in \mathbb{N}_{0}:=\mathbb{N} \cup\{0\} ; \mathbb{N}:=\{1,2,3, \ldots\}\right) .
\end{aligned}
$$

Note that, by comparing definitions (1.5), (1.6), and (6.1), we can deduce the following connections:

$$
\Omega_{0}(\kappa)=\frac{k \sqrt{2}}{\kappa} K(k) \quad \text { and } \quad \Omega_{1}(\kappa)=\frac{k \sqrt{2}}{\kappa\left(1-\kappa^{2}\right)} E(k) \quad\left(k^{2}:=\frac{2 \kappa^{2}}{1+\kappa^{2}}\right) .
$$


These Epstein-Hubbell elliptic-type integrals can be extended by using definitions, which we have considered in this paper, can be generalized by introducing $(p, q)$-extended Epstein-Hubbell elliptic-type integral in (6.1):

$$
\begin{aligned}
& \Omega_{\gamma, p, q}(\kappa):=\int_{0}^{\pi} \frac{1}{\left(1-\kappa^{2} \cos \theta\right)^{\gamma+\frac{1}{2}}} \mathrm{e}^{-\frac{p}{\sin ^{2}(\theta / 2)}-\frac{q}{\cos ^{2}(\theta / 2)}} \mathrm{d} \theta \\
& \left(\min \{\Re(p), \Re(q)\}>0 ; 0 \leqq \kappa \leqq 1 ; \gamma \in \mathbb{C}_{0} \text { when } p=q=0\right) .
\end{aligned}
$$

It is worth noting that if we substitute $t=\cos \left(\frac{\theta}{2}\right)=\sqrt{\frac{(1+\cos \theta)}{2}}$ in (2.4), we obtain the following connection with the $(p, q)$-extended elliptic-type integrals $\mathcal{H}_{p, q}(k, \gamma)$ defined by (2.4) and $\Omega_{\gamma, p, q}(k)$ defined by $(6.2)$

$$
\mathcal{H}_{p, q}(k, \gamma)=\frac{\left(2-k^{2}\right)^{\gamma-\frac{1}{2}}}{2^{\gamma+\frac{1}{2}}} \Omega_{-\gamma, p, q}\left(\frac{k}{\sqrt{2-k^{2}}}\right) .
$$

Again by letting $\cos (\theta / 2)=t$ and then $t^{2}=u$ in (6.2), we can easily obtain relationship (6.3) in terms of $(p, q)$-extended Gauss' hypergeometric function (1.8)

$$
\Omega_{\gamma, p, q}(\kappa):=\frac{\pi}{\left(1+\kappa^{2}\right)^{\gamma+\frac{1}{2}}} F_{p, q}\left(\gamma+\frac{1}{2}, \frac{1}{2} ; 1 ; \frac{2 \kappa^{2}}{1+\kappa^{2}}\right) .
$$

Note that expression (6.3) for $p=q=0$ reduces to the known result [37]. Further many of properties such as Mellin transform formulas, infinite series representations containing Laguerre polynomials, log-convexity property, and Turán-type inequalities and differentiation and integral formulas can be easily derived. As a result, the information involved can be left as an exercise for the curious reader. More various concavity, convexity, and monotonicity properties of our findings can be studied and investigated parallel to the recent papers $[1,28,36,38,39]$.

\section{Acknowledgements}

The present research work of the first author is carried out under the project MATRICS, SERB, Department of Science \& Technology (DST), India (File No. MTR/2019/001328). Moreover, the authors thank the referees for their concrete suggestions which resulted in a better organization of this article.

\section{Funding}

Not available.

Availability of data and materials

No data were used to support this study.

Competing interests

The authors declare that they have no competing interests.

\section{Authors' contributions}

The authors contributed equally and significantly in writing this paper. All authors read and approved the final manuscript.

\section{Author details}

'Department of HEAS (Mathematics), University College of Engineering and Technology, Bikaner, 334004, Rajasthan, India. ${ }^{2}$ Department of Mathematics, Malaviya National Institute of Technology, Jaipur, 302017, India. ${ }^{3}$ Department of HEAS (Mathematics), Rajasthan Technical University, Kota, 324010, India.

\section{Publisher's Note}

Springer Nature remains neutral with regard to jurisdictional claims in published maps and institutional affiliations. 


\section{References}

1. Alzer, H., Richards, K.C.: A concavity property of the complete elliptic integral of the first kind. Integral Transforms Spec. Funct. 31(9), 758-768 (2020)

2. Anderson, G.D., Vamanamurthy, M.K., Vuorinen, M.: Conformal Invariants, Inequalities, and Quasiconformal Maps. Wiley, New York (1997)

3. Barton, G.: Do attractive scattering potentials concentrate particles at the origin in one, two and three dimensions? III: high energies in quantum mechanics. Proc. R. Soc. Lond. Ser. A 388, 445-456 (1983)

4. Björkberg, J., Kristensson, G.: Electromagnetic scattering by a perfectly conducting elliptic disk. Can. J. Phys. 65 , 723-734 (1987)

5. Bushell, P.J.: On a generalization of Barton's integral and related integrals of complete elliptic integrals. Math. Proc Camb. Philos. Soc. 101, 1-5 (1987)

6. Byrd, P.F., Friedman, M.D.: Handbook of Elliptic Integrals for Engineers and Scientists. Springer, Berlin (1971)

7. Carlson, B.C.: Some series and bounds for incomplete elliptic integrals. J. Math. Phys. 40, 125-134 (1961)

8. Chaudhry, M.A., Qadir, A., Raflque, M., Zubair, S.M.: Extension of Euler's beta function. J. Comput. Appl. Math. 78, 19-32 (1997)

9. Chaudhry, M.A., Qadir, A., Srivastava, H.M., Paris, R.B.: Extended hypergeometric and confluent hypergeometric functions. Appl. Math. Comput. 159, 589-602 (2004)

10. Choi, J., Parmar, R.K., Pogány, T.K.: Mathieu-type series built by $(p, q)$-extended Gaussian hypergeometric function. Bull. Korean Math. Soc. 54(3), 789-797 (2017)

11. Choi, J., Rathie, A.K., Parmar, R.K.: Extension of extended beta, hypergeometric and confluent hypergeometric functions. Honam Math. J. 36, 339-367 (2014)

12. Epstein, L.F., Hubbell, J.H.: Evaluation of a generalized elliptic-type integral. J. Res. Natl. Bur. Stand. B, Math. Math. Phys. 67, 1-17 (1963)

13. Erdélyi, A., Magnus, W., Oberhettinger, F., Tricomi, F.G.: Higher Transcendental Functions, Volume I. McGraw-Hill, New York (1953)

14. Hubbell, J.H., Bach, R., Lamkin, J.: Radiation field from a rectangular source. J. Res. Natl. Bur. Stand. C, Eng. Instrum. 64C(2), 121-138 (1960)

15. Kristensson, G.: Acoustic scattering by a soft elliptic disk. J. Sound Vib. 103, 487-498 (1985)

16. Lin, S.-D., Chang, L.-F., Srivastava, H.M.: A certain class of incomplete elliptic integrals and associated definite integrals. Appl. Math. Comput. 215, 1176-1184 (2009)

17. Luo, M.J., Parmar, R.K., Raina, R.K.: On extended Hurwitz-Lerch zeta function. J. Math. Anal. Appl. 448, 1281-1304 (2017)

18. Maširević, D.J., Parmar, R.K., Pogány, T.K.: $(p, q)$-extended Bessel and modified Bessel functions of the first kind. Results Math. 72(1-2), 617-632 (2017)

19. Paris, R.B., Kaminski, D.: Asymptotics and Mellin-Barnes Integrals. Cambridge University Press, Cambridge (2001)

20. Parmar, R.K., Chopra, P., Paris, R.B.: On an extension of extended beta and hypergeometric functions. J. Class. Anal. 11(2), 91-106 (2017)

21. Parmar, R.K., Pogány, T.K.: Extended Srivastava's triple hypergeometric $H_{A p, q}$ function and related bounding inequalities. J. Contemp. Math. Anal. 52(6), 261-272 (2017)

22. Parmar, R.K., Pogány, T.K.: On $(p, q)$-extension of further members of Bessel-Struve functions class. Miskolc Math. Notes 20, 451-463 (2019)

23. Parmar, R.K., Pogany, T.K.: On Mathieu-type series for the unified Gaussian hypergeometric functions. Appl. Anal. Discrete Math. 14(1), 138-149 (2020)

24. Parmar, R.K., Pogány, T.K., Saxena, R.K.: On properties and applications of $(p, q)$-extended $\tau$-hypergeometric functions. C. R. Math. 356(3), 278-282 (2018)

25. Prudnikov, A.P., Brychkov, Yu.A., Marichev, O.I.: Integrals and Series, Volume II: Special Functions. Nauka, Moscow (1983) (In Russian); Translated from the Russian by, Gould, G. G., Gordon and Breach Science Publishers, New York, Philadelphia, London, Paris, Montreux, Tokyo and Melbourne, 1986

26. Prudnikov, A.P., Brychkov, Yu.A., Marichev, O.I.: Integrals and Series, Volume III: More Special Functions. Nauka, Moscow (1986) (In Russian); Translated from the Russian by, Gould, G. G., Gordon and Breach Science Publishers, New York, Philadelphia, London, Paris, Montreux, Tokyo and Melbourne, 1990

27. Rainville, E.D.: Special Functions. Macmillan, New York (1960) Reprinted by Chelsea Publishing Company, Bronx, New York, 1971

28. Richards, K.C., Smith, J.N.: A concavity property of generalized complete elliptic integrals. Integral Transforms Spec. Funct. 32(3), 240-252 (2021)

29. Srivastava, H.M.: Some elliptic integrals of Barton and Bushell. J. Phys. A, Math. Gen. 28, 2305-2312 (1995)

30. Srivastava, H.M., Bromberg, S.: Some families of generalized elliptic-type integrals. Math. Comput. Model. 21(3), 29-38 (1995)

31. Srivastava, H.M., Gupta, K.C., Goyal, S.P.: The H-Functions of One and Two Variables with Applications. South Asian Publishers, New Delhi (1982)

32. Srivastava, H.M., Karlsson, P.W.: Multiple Gaussian Hypergeometric Series. Halsted, Chichester (1985)

33. Srivastava, H.M., Parmar, R.K., Chopra, P.: Some families of generalized complete and incomplete elliptic-type integrals. J. Nonlinear Sci. Appl. 10, 1162-1182 (2017)

34. Srivastava, H.M., Siddiqi, R.N.: A unified presentation of certain families of elliptic-type integrals related to radiation field problems. Radiat. Phys. Chem. 46, 303-315 (1995)

35. Srivastava, R.: A note on the Epstein-Hubbell generalized elliptic-type integral. Appl. Math. Comput. 69, 255-262 (1995)

36. Wang, M., Zhang, W., Chu, Y.: Monotonicity, convexity and inequalities involving the generalized elliptic integrals. Acta Math. Sci. 39, 1440-1450 (2019)

37. Weiss, G.H.: A note generalized elliptic integral. J. Res. Natl. Bur. Stand. B, Math. Math. Phys. 68, 1-2 (1964)

38. Yang, Z.-H., Qian, W.-M., Chu, Y.-M., Zhang, W.: On approximating the arithmetic-geometric mean and complete elliptic integral of the first kind. J. Math. Anal. Appl. 462(2), 1714-1726 (2018)

39. Zhang, X.: Monotonicity and functional inequalities for the complete $p$-elliptic integrals. J. Math. Anal. Appl. 453(2) 942-953 (2017) 